\title{
Free energies of iron phases at high pressure and temperature: Molecular dynamics study
}

\author{
Anatoly B. Belonoshko $\odot,{ }^{1,2}$ Jie Fu $\odot,{ }^{3}$ and Grigory Smirnov $\odot^{2,4}$ \\ ${ }^{1}$ Condensed Matter Theory, Department of Physics, AlbaNova University Center, Royal Institute of Technology (KTH), \\ 10691 Stockholm, Sweden \\ ${ }^{2}$ National Research University Higher School of Economics, 123458 Moscow, Russia \\ ${ }^{3}$ Department of Physics, School of Physical Science and Technology, Ningbo University, 315211 Ningbo, China \\ ${ }^{4}$ Joint Institute for High Temperatures of the Russian Academy of Sciences, 125412 Moscow, Russia
}

(Received 10 June 2021; accepted 25 August 2021; published 9 September 2021)

\begin{abstract}
The crystal structure of iron, the major component of the Earth's inner core (IC), is unknown under the IC high pressure (P) (3.3-3.6 Mbar) and temperature (T) (5000-7000 K). Experimental and theoretical data on the phase diagram of iron at these extreme PT conditions are contradictory. Applying quasi-ab initio and $a b$ initio molecular dynamics we computed free energies of the body-centered cubic $(b c c)$, hexagonal close-packed ( $h c p$ ), and liquid phases. The ionic free energies, computed for the embedded-atom model, were corrected for electronic entropy. Such correction brings the melting temperatures of the $h c p$ iron in very good agreement with previous $a b$ initio data. This validates the calculation of the $b c c$ phase, where fully $a b$ initio treatment is not technically possible due to large sizes required for convergence. The resulting phase diagram shows stabilization of the $b c c$ phase prior to melting in the pressure range of the IC. The melting temperature of the $b c c$ phase is equal to $7190 \mathrm{~K}$ at the pressure $360 \mathrm{GPa}$.
\end{abstract}

DOI: 10.1103/PhysRevB.104.104103

\section{INTRODUCTION}

It has been established that the Earth's inner core (IC) mainly consists of iron [1-5]. It has been assumed that $\mathrm{Fe}$ is stable at the IC pressure (P) (3.3-3.6 Mbar) and temperature $(\mathrm{T})(5000-7000 \mathrm{~K})$ in the hexagonal close-packed (hcp) phase, likely alloyed with $\mathrm{Ni}$ and light elements [5]. This assumption, of course, has some ground - the hexagonal close-packed ( $h c p$ ) phase of $\mathrm{Fe}$ was demonstrated to be stable at room temperature up to the pressure of $3 \mathrm{Mbar}$ [5]. The impact of temperature has appeared to be rather controversial. A number of phases have been suggested in addition to face-centered cubic $(f c c)$, body-centered cubic $(b c c), h c p$, and liquid. Namely, the orthorhombic phase, the double hcp phase, the $f c c$ phase outside of the "orthodox" PT field of its stability, and finally, the high-PT nonmagnetic $b c c$ phase. The general consensus, based on the observation of the hcp [6-9] in a broad-PT range (but not quite at the IC PT conditions) is that the $h c p$ phase is stable in the IC.

However, a number of theoretical [10-19] and experimental [14,20-25] studies demonstrate that this might not be the case. Particularly strong evidence in support of the $b c c$ phase is that the $h c p$ phase becomes isotropic at the PT of the IC $[11,26]$ and, thus, is incapable to account for the

Published by the American Physical Society under the terms of the Creative Commons Attribution 4.0 International license. Further distribution of this work must maintain attribution to the author(s) and the published article's title, journal citation, and DOI. Funded by Bibsam. anisotropy of the IC [27]. There is widespread confusion that the $h c p$ is anisotropic and that confusion is based on the paper by Steinle-Neumann and co-authors [28]. The results of that paper have been demonstrated to be incorrect [11]. Steinle-Neumann and co-authors [28] later confirmed that the results on anisotropy were wrong and that the anisotropy of the $h c p$ iron under core conditions is less than $1 \%$ [26,29], well below the anisotropy in the Earth's IC. Another paper that supports the anisotropic nature of the $h c p$ phase is the work by Antonangeli et al. [30]. In that paper, the elastic data were obtained at rather moderate pressures and rather low temperatures compared with the PT in the IC. Then the data were extrapolated ad hoc to the IC conditions, the theory being in reasonable agreement with the experiment predicts isotropic $h c p$ at the IC conditions. Such features as strong attenuation and low shear modulus of the material in the IC are at odds with the high shear modulus of the $h c p$ phase. On the contrary, the $b c c$ phase has low shear modulus [12] and is strongly attenuating material due to its uniquely low viscosity [19].

Finally, a very recent paper [31] shows that the innermost IC has anisotropy where the fast and slow directions of seismic signal propagation cross at the angle $54^{\circ}$. This is the exact match to the fast and slow directions of sound propagation in the $b c c$ phase [13,32]. Namely, the fast (111) and slow (100) directions in the $b c c$ cross at the angle $54.73^{\circ}$-the exact match to the seismic data. This match is of paramount importance - the Earth itself tells us what kind of material is inside its core.

Therefore, it is not a question anymore of what phase of iron is stable in the Earth IC. Rather, it is a question of what makes it so difficult to observe the $b c c$ phase in experiments. 
In this paper, by computing free energies of iron phases, we determine the PT field of stability of the $b c c$ phase, in particular, the $h c p-b c c$ phase boundary and the melting temperatures of the $b c c$ phase.

\section{METHODS}

To compute the free energies of the iron phases, we performed ab initio and classical molecular dynamics (AIMD and $\mathrm{MD}$, correspondingly) simulations of the $b c c, h c p$, and liquid phases of iron under the IC PT and beyond conditions.

The reason for such a combination (AIMD+MD) is the following. The $b c c$ phase is dynamically unstable at low temperature. Its dynamical stability is due to the high temperature that enables superioniclike diffusion $[18,19]$. The mechanism of the stabilization is common both for DFT [18] and for the embedded-atom method (EAM) $[10,11]$ description of interactions. The onset of the diffusion is forbidden in too small computational cells because of the oversymmetrization due to periodic boundary conditions (PBC). While the calculation of the forces is not affected by small sizes, the correlated motion that involves several crystal planes is affected by the small size of the cell due to imposed periodicity. Therefore, for correct modeling of the $b c c$ phase one needs a rather large (and depending on PT conditions at times very large) number of particles involved in the simulation. This makes application of the DFT method prohibitively expensive even for single runs. For the calculations of free energies it becomes quite impractical. It was demonstrated that the thermodynamic stabilization of $b c c$ on temperature increase at the pressure in the center of the Earth is due to electronic entropy [18]. However, there are no electrons in the EAM description. The electrons are implicitly there affecting the interaction of atoms; however, the substantial part of the entropy is lost in the EAM description because of the significant reduction in the number of degrees of freedom. One needs to estimate the electronic entropy independently. Therefore, the strategy of the calculations in this paper is to compute the free energies using the EAM model and then correct them by the electronic entropy computed from first principles.

In our DFT-based MD runs we used the generalizedgradient approximation of the electronic exchange-correlation energy [33] and the $\mathrm{Fe}$ potential with $3 p^{6} 3 d^{6} 4 s^{2}$ valence electrons. Electronic structure calculations [34-36] were performed with a plane-wave set corresponding to a $350.0 \mathrm{eV}$ energy cutoff and the electronic iterations were converged to within $10^{-4} \mathrm{eV}$. We used a 1.0-fs time step for the AIMD calculations and the total energy of the electron-ion system was conserved with an accuracy of $8 \mathrm{meV} / \mathrm{ps}$. We have approximated the $h c p$ and $b c c$ phases by supercells with sizes ranging from 432 atoms $(6 \times 6 \times 6$ unit cells $)$ for the $h c p$ structure to 1024 atoms $(8 \times 8 \times 8$ cubic unit cells). Liquid phase was obtained by heating the hcp phase up to a very high temperature and then equilibrating it at the temperatures around melting.

The structure of phases was monitored by calculating radial distribution function (RDF) and diffusion. We ran the AIMD calculations for all three phases in the NPT ensemble with the Nosé thermostat and Parrinello-Rahman barostat. The AIMD runs duration were at least 4000 time steps and up to 18,000 time steps. The electronic entropy for all three phases was computed at two pressures, namely, 120 and $360 \mathrm{GPa}$ at the temperatures 4000 and $7000 \mathrm{~K}$, correspondingly. The AIMD runs have been performed for $\Gamma$-point only. At the sizes of the supercells that we use, the $\Gamma$-point is quite sufficient. In all our AIMD runs, we used the VASP $[35,36]$ software package.

The EAM MD runs were performed using the EAM potential developed without reference to experimental data, the so-called quasi-ab initio [10]. The potential was fitted to the energies of a number of iron configurations including liquidlike at zero temperature computed by the full potential linear muffin-tin orbital (FPLMTO) method. The EAM is in the Sutton-Chen [37] format fitted to FPLMTO energy-volume data for $h c p$ and liquid iron [10]. The total potential energy of a configuration is

$$
E_{\text {conf }}=\sum_{i=1}^{N} E_{i},
$$

where $N$ is the number of atoms and $E_{i}$ is the potential energy of atom $i$. The Sutton-Chen potential includes two parts: One part is a pairwise potential term, and the other part is the term of embedding energy that is the energy required to place an atom into an electron cloud. Its particular form is as follows:

$$
E_{i}=\frac{1}{2} \sum_{j=1, j \neq i}^{N} \varphi\left(r_{i j}\right)+F\left(\rho_{i}\right),
$$

with

$$
\begin{gathered}
\varphi\left(r_{i j}\right)=\epsilon\left(\frac{a}{r_{i j}}\right)^{n}, \\
F\left(\rho_{i}\right)=-\epsilon C \sqrt{\rho_{i}}, \\
\rho_{i}=\sum_{j=1, j \neq i}^{N}\left(\frac{a}{r_{i j}}\right)^{m},
\end{gathered}
$$

where $r_{i j}$ is the distance between atom $i$ and atom $j, \varphi(r)$ is the pairwise potential between atom $i$ and $j, F(\rho)$ is the embedding energy function, and $\rho_{i}$ is the sum of contribution of electron charge density of $j$ sites atoms at the location of $i$ site. As a result of the fit, the parameters are $n=8.137$, $m=4.788, \epsilon=0.0173, a=3.4714 \AA$, and $C=24.939$. The potential was demonstrated to perform well at high pressures in close agreement with DFT results [10]. Even though the $b c c$ configurations have not been used for fitting, the dynamic stability dependence on size calculated for the EAM and DFT models is identical for the sizes available to ab initio MD [18].

Using this potential we computed a number of properties of $h c p, b c c$, and liquid phases including free energies. Since the EAM-based MD are very fast, the calculations were performed on large samples (up to 16,000,000 atoms) for long times (up to 1,000,000 time steps). The same thermostat and barostat as well as the time step as for the AIMD have been used. We also ran NVT EAM MD runs and also used Andersen thermostat. All the EAM-based MD runs were performed using LAMMPS software package [38].

We calculated the absolute free energies of liquid and solid phases using nonequilibrium (NE) MD, which is implemented in the LAMMPS package $[39,40]$. In the NE method, the 
coupling parameter $\lambda=\lambda(t)$ changes continuously during the simulation to drive a system from an initial state $(i)$ to a final state $(f)$.

It is assumed that the free energy of the final state is known analytically or numerically. Then, the Hamiltonian interpolation procedure allows for determining the free-energy difference between the two states and the absolute free energy of the initial state. In this approach the potential energy function $U$ is parameterized to construct the linear interpolation between two states:

$$
U(\lambda)=(1-\lambda) U_{i}+\lambda U_{f},
$$

where $U_{i}$ is the system of interest interaction potential and $U_{f}$ is the reference model with the known free energy.

Setting $\lambda_{i}=0$ and $\lambda_{f}=1$, respectively, the irreversible work during the switching procedure in a time $t_{s}$ is determined as

$$
W_{i-f}^{\mathrm{irr}}=\int_{0}^{t_{s}} \frac{d \lambda}{d t}\left(U_{f}-U_{i}\right) d t .
$$

The free-energy difference between two states $\Delta F$ could be accurately determined by conducting multiple simulations in both directions,

$$
\Delta F=\frac{1}{2}\left(\bar{W}_{i-f}^{\mathrm{irr}}-\bar{W}_{f-i}^{\mathrm{irr}}\right),
$$

where $\bar{W}_{i-f}^{\mathrm{irr}}$ and $\bar{W}_{f-i}^{\mathrm{irr}}$ are the mean value over independent switching simulations for the forward and backward directions correspondingly.

In practice, the integral in Eq. (7) is calculated in terms of the sum

$$
W_{i-f}^{\mathrm{irr}}=\sum_{n}^{N} \Delta \lambda_{n}\left(U_{f}-U_{i}\right)_{n \Delta t},
$$

where $\Delta t$ is the time step, the index $n$ is the number of time step, and $N$ is the total number of time steps.

Having determined the free-energy difference, the absolute Helmholtz and Gibbs free energies of a physical system are determined from

$$
\begin{gathered}
F_{\mathrm{syst}}\left(V_{0}, T_{0}\right)=F_{f}\left(V_{0}, T_{0}\right)+\frac{1}{2}\left(\bar{W}_{i-f}^{\mathrm{irr}}-\bar{W}_{f-i}^{\mathrm{irr}}\right) \\
G_{\text {syst }}\left(P_{0}, T_{0}\right)=F_{\text {syst }}\left(V_{0}, T_{0}\right)+P_{0} V_{0},
\end{gathered}
$$

where $V_{0}$ is the equilibrium volume at the given $P=P_{0}$ and $T=T_{0}$.

We use the Einstein crystal as the reference for the free energy of a solid phase:

$$
F_{f}^{\mathrm{sol}}\left(V_{0}, T_{0}\right)=3 N k_{b} T \ln \left(\frac{\hbar \omega}{k_{b} T_{0}}\right) .
$$

The frequency $\omega$ is determined from mean-square displacement (MSD) calculations at the each volume and temperature:

$$
\omega\left(V_{0}, T_{0}\right)=\sqrt{\frac{3 k_{b} T_{0}}{m\left\langle\Delta r^{2}\right\rangle_{V_{0}, T_{0}}}} .
$$

The Uhlenbeck-Ford (UF) model is used as the reference for the calculation of the free energy of the liquid phase. It is defined by the interatomic pair potential

$$
U_{\mathrm{UF}}(r)=-p k_{b} T \ln \left(1-e^{(-r / \sigma)^{2}}\right),
$$

where $\sigma$ is a length-scale parameter, and $p>0$ is a scaling factor [41].

The UF fluid is thermodynamically stable for; a set of accurate numerical cubic-spline approximations is available to determine the excess free energy of this model [42].

A similar procedure can be used to construct the temperature dependence of the Gibbs free energy $G\left(P_{0}, T\right)$ at finite pressure $P=P_{0}$. Scaling the potential energy $U\left(\boldsymbol{r}_{1}, \ldots, \boldsymbol{r}_{N}\right)$ by the coupling parameter $\lambda$, the configurational part of the partition function $Z_{R S}(\lambda)$ at pressure $P(\lambda)$ and temperature $T_{0}$ is given by

$$
Z_{R S}(\lambda)=\int_{0}^{\infty} d V \exp \left[-\frac{P(\lambda) V}{k_{b} T_{0}}\right] \int_{V} d^{3 N} \boldsymbol{r} \exp \left(-\frac{\lambda U}{k_{b} T_{0}}\right) .
$$

Introducing the scaling relation $T=T_{0} / \lambda$, it can be shown that the Gibbs free energy of the scaled and physical system is related according to

$$
G_{\text {syst }}(T)=\frac{G_{R S}(\lambda)}{\lambda}+\frac{3}{2} N k_{b} T_{0} \frac{\ln \lambda}{\lambda} .
$$

The function $G_{R S}(\lambda)$ is evaluated with the NE technique, where $\lambda(t)$ and $P(\lambda(t))$ are varied dynamically at a fixed temperature $T_{0}$. The parameter $(\lambda)$ varies from $\lambda(0)=1$ to $\lambda\left(t_{s}\right)=\lambda_{f}$. The final value $\lambda_{f}$ determines the temperature range of Gibbs free energy. Assuming the linear scaling of pressure $P=\lambda(t) P_{0}$, the corresponding irreversible work is calculated from [43]

$$
\begin{aligned}
W_{1-f}^{\mathrm{irr}} & =\int_{0}^{t_{s}} \frac{d \lambda}{d t}\left(U(t)+\frac{d P}{d \lambda} V(t)\right) d t \\
& =\int_{0}^{t_{s}}\left(U(t)+P_{0} V(t)\right) d t .
\end{aligned}
$$

After repeating the procedure in both directions multiple times, we obtain the temperature dependence of Gibbs free energy along the isobar $P=P_{0}$ :

$$
\begin{aligned}
G_{\text {syst }}\left(P_{0}, T\right)= & \frac{G_{R S}\left(P_{0}, T_{0}\right)}{\lambda}+\frac{3}{2} N k_{b} T_{0} \frac{\ln \lambda}{\lambda} \\
& +\frac{1}{2 \lambda}\left(\bar{W}_{1-f}^{\mathrm{irr}}-\bar{W}_{f-1}^{\mathrm{irr}}\right) .
\end{aligned}
$$

The simulations of $b c c$ solid were carried out in a cubic box with $18 \times 18 \times 18 b c c$ unit cells (11,664 atoms). The same system is used for liquid simulations.

To determine the melting temperature $T_{m}$ at a given pressure $P_{0}$ we use the following procedure. At the first step the equilibrium densities of $b c c$ and $h c p$ solids are calculated at a temperature $T_{1}$ below the estimated melting point $T_{m}$. We determine the average value over a 1-ns MD trajectory, obtained with constant pressure-temperature simulation. The same procedure is repeated for liquid at a temperature $T_{2}$ above the estimated melting point.

At the next step, the nonequilibrium Hamiltonian interpolation technique is used to determine the absolute values of the Gibbs free energy of $b c c G_{b c c}\left(P_{0}, T_{1}\right), h c p G_{h c p}\left(P_{0}, T_{1}\right)$, and liquid $G_{\mathrm{liq}}\left(P_{0}, T_{2}\right)$. We perform 10 independent NVT MD simulations in forward and backward directions. Each run starts 
from the equilibration part during $0.1 \mathrm{~ns}$, then the interaction potential is switched during $0.5 \mathrm{~ns}$. The Langevin thermostat is used to control the temperature. The UF fluid with $p=75$ and $\sigma=1.2$ is used as a reference for liquid simulations.

Finally, the RS method is used to construct temperature dependence of the Gibbs free energy $G_{b c c}\left(P_{0}, T\right)$ and $G_{\text {liq }}\left(P_{0}, T\right)$.

\section{RESULTS}

It has been demonstrated that properties of the $b c c$ phase are strongly size dependent $[18,19]$. However, that observation was objected [44]. The authors wrote that they computed free energies of the $b c c$ phase and found the free energy to be constant while changing the size of the cell from 1024 atoms $(8 \times 8 \times 8$ unit cells $)$ to 65,536 atoms $(32 \times 32 \times 32$ unit cells). This result was obtained for the free energy computed at the pressure of $360 \mathrm{GPa}$ and temperature $7000 \mathrm{~K}$. We have performed the simulations in the NPT ensemble at the same PT parameters and the EAM potential that was used by the authors [44]. We observed that at these conditions the $b c c$ structure was not preserved. Instead, the $b c c$ phase melted and the RDFs for both sizes [Fig. 1(a)] are typical for the liquid state. Figure 1(b) shows MSD computed for 1024 and 65,536 atoms. They are also almost identical and typical of the liquid state. That means that all the results obtained by the authors [44] are relevant for the liquid state. Indeed, the properties of the liquid metal converge at very small sizes. Normally, computational cells containing a hundred and more atoms are quite sufficient for calculation of thermodynamic properties. Therefore, it is only natural that the authors did not see any changes when computing the free energy with 1024 and 65,536 atoms. They simply computed it for the liquid (or superheated states if the runs have been too short). We note that the authors used the Anderson thermostat in their simulations. The thermostat requires randomization of velocities with some period. That period was set by the authors equal to 5000 time steps. We computed MSD for the 65,536 atoms applying both Nosé thermostat and Anderson thermostat. We used the EAM potential [19] that preserves the $b c c$ phase at the $\mathrm{P}=360 \mathrm{GPa}$ and $\mathrm{T}$ $=7000 \mathrm{~K}$. One can see (Fig. 2) that the MSD is substantially higher for the Nosé thermostat. The reason is the following. Atoms in the $b c c$ are in a superionic state. The motion is highly correlated and it takes time for atoms to move in a correlated way. The Anderson thermostat destroys that correlation by periodic randomization of correlated velocities. That decreases the diffusion (Fig. 2). Such decrease might restrict the number of available states (or, at least, increase the time needed for simulation) and, correspondingly, the entropy that is proportional to the logarithm of the visited states. Thus, we can conclude that the paper [44] did not study the $b c c$ phase and even if it would have, the thermostat they used [44] is not suitable for simulating a superionic state. The thermostat provides the data on diffusion in substantial disagreement with the thermostat known to provide correct thermodynamics. We can safely conclude that the results obtained by Schultz and co-authors [44] are not relevant for the $b c c$ phase and the method they used is not suitable for the study of the $b c c$ phase.

However, the question remains: What is the size dependence of the thermodynamic properties in general and free

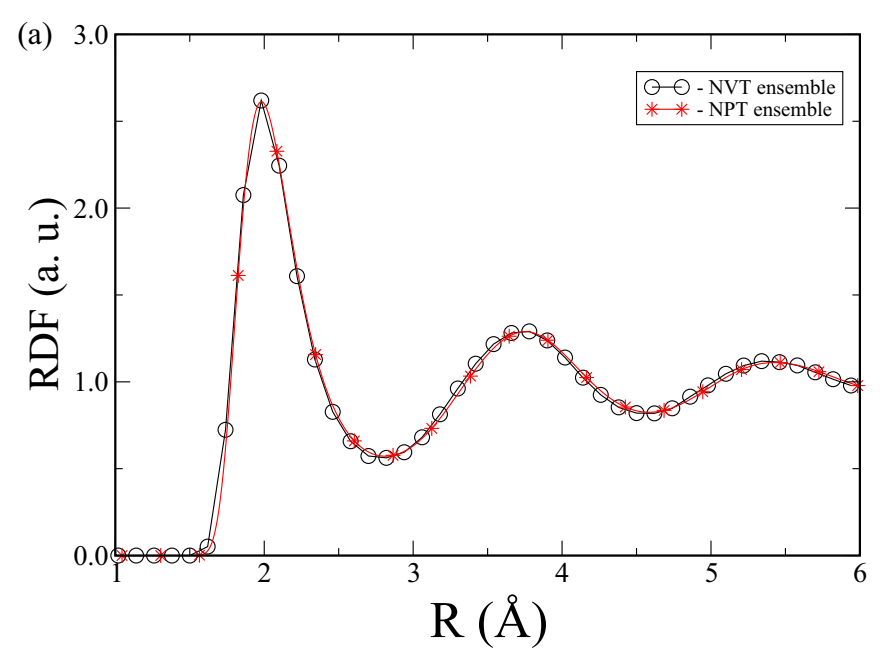

(b)

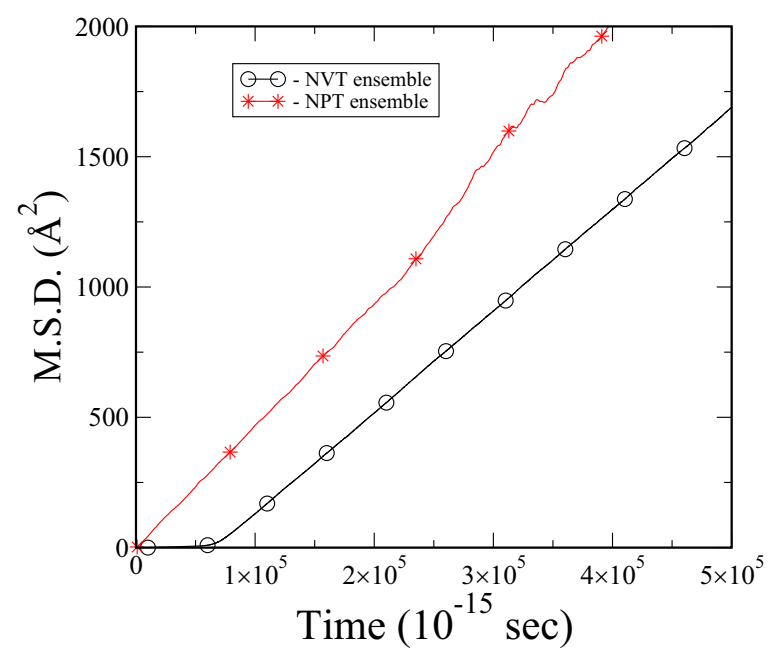

FIG. 1. RDF (a) and MSD (b) calculated with the EAM as applied in Ref. [44]. Both RDF and MSD in Ref. [44] are calculated using NVT ensemble with the Andersson thermostat, while results in this paper are computed with Nosé thermostat. The RDFs and MSD are nearly identical. The MSD from the EAM in Ref. [44] is typical of solid for nearly 100,000 time steps and then is typical of liquid state. The slope of MSDs is the same. This suggests that Ref. [44] describes the data obtained for the liquid state.

energy of the $b c c$ phase in particular? We performed simulations of the $b c c$ phase at $7000 \mathrm{~K}$ and $360 \mathrm{GPa}$ in a wide range of sizes, from 432 to 2 million atoms. Figure 3 shows the RDFs of the calculated samples. The $b c c$ phase preserves its structure starting from 1024 atoms. From that size and above one can see only subtle changes in RDF (Fig. 3). When the size of the system is under 1024 atoms, that is less than $8 \times 8 \times 8$ unit cells, the $b c c$ structure collapses into the $h c p$ structure. Remarkably, this is exactly the result we observe in DFT MD simulations [18]. This suggests that the dynamics of $\mathrm{Fe}$ atoms in the EAM and DFT description are very similar. The dynamics of $\mathrm{Fe}$ atoms in the $b c c$ structure is very special. Atoms are diffusing like in liquid, yet preserving all the properties of a solid $[12,13]$. Figure 4 shows the MSD size dependence computed at $7000 \mathrm{~K}$ and $360 \mathrm{GPa}$. We note that while the $b c c$ structure is preserved already at the size $8 \times 8 \times 8$ unit cells, the MSD is not converged until there 


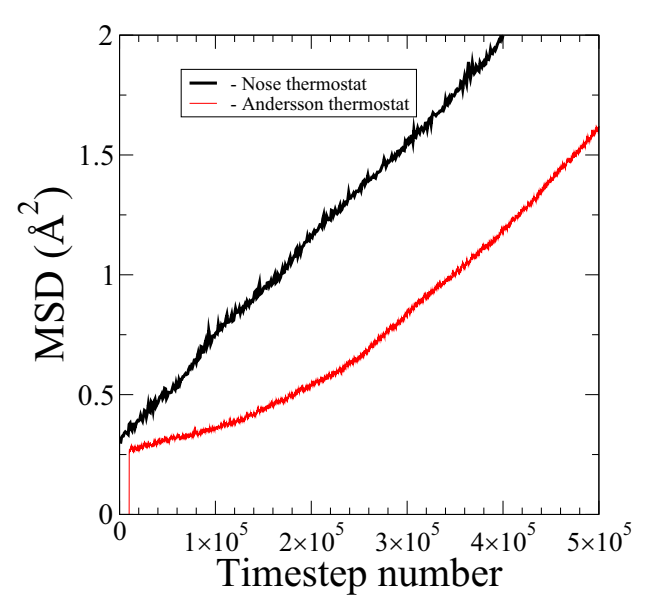

FIG. 2. Comparison of the MSD in $b c c$ structure computed with Nosé and Andersson thermostat. The MD simulations performed for the EAM potential in Ref. [10]. The Andersson thermostat provides considerably smaller MSD because of velocities randomization every 5000 time steps. This randomization destroys highly correlated motion and should not be used. On large times the slope of the MSD becomes equal.

are much larger sizes. Therefore, the $8 \times 8 \times 8$ size is the minimum required for computing the $b c c$ phase properties. This invalidates all the previous MD simulations of the $b c c$ phase performed at smaller (and at times much smaller) sizes than 1024 atoms.

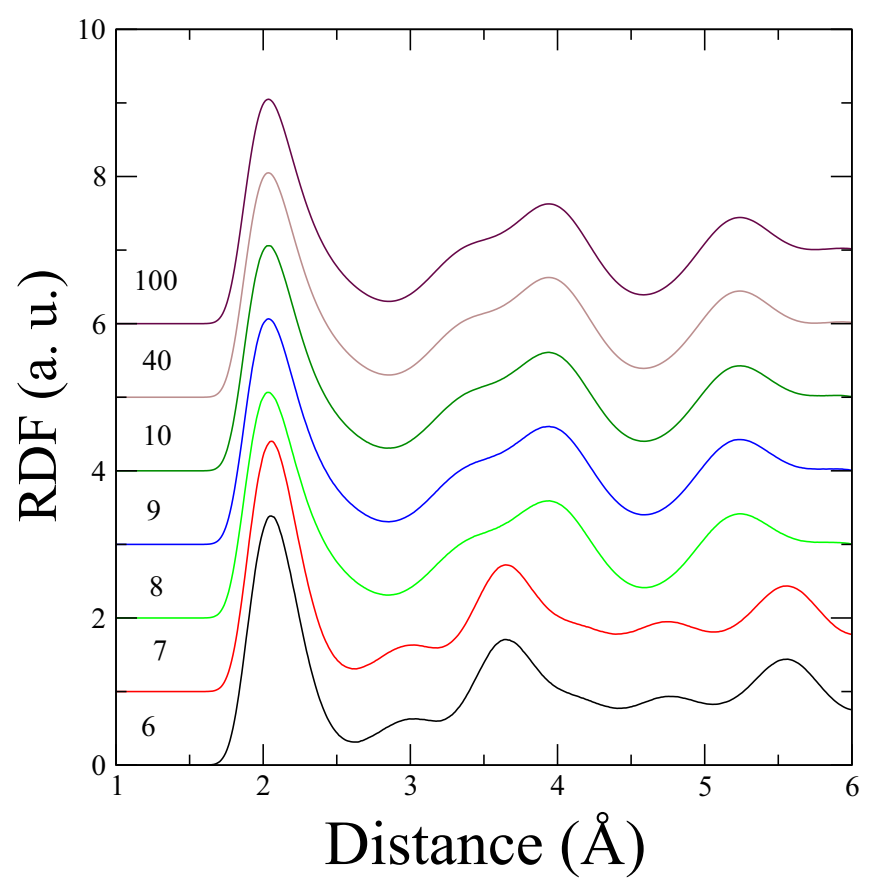

FIG. 3. RDFs of $b c c$ iron at $\mathrm{P}=360 \mathrm{GPa}$ and $\mathrm{T}=7000 \mathrm{~K}$. Calculations performed at the sizes indicated on the legend. At the sizes below 1024 atoms the structure collapses to close-packed phase while at the higher sizes the structure remains $b c c$. The number $\mathrm{n}$ means the simulation was performed for the computational cell with $n \times n \times n$ unit cells, where the unit cell contains two atoms.

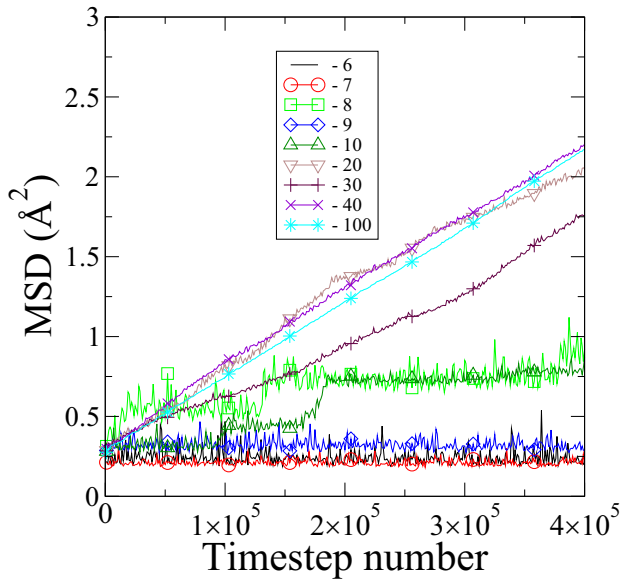

FIG. 4. MSD in $b c c$ iron at $\mathrm{P}=360 \mathrm{GPa}$ and $\mathrm{T}=7000 \mathrm{~K}$. The MSD converges at about 100,000 atoms; still, certain differences remain up to the highest size. The number $\mathrm{n}$ means the simulation was performed for the computational cell with $n \times n \times n$ unit cell, where the unit cell contains two atoms.

We have also studied the limits of dynamic stability on decreasing temperature at the same pressure of $360 \mathrm{GPa}$. The cell with size 2,000,000 atoms $(100 \times 100 \times 100$ unit cells $)$ is dynamically stable down to the temperature of $3590 \mathrm{~K}$. However, the cell with the size $1,024,000(80 \times 80 \times 80$ unit cells) transforms to the $h c p$ structure. This means that detailed studies of the iron phase diagram are not technically possible at present on the basis of DFT methods alone. The observed extreme size dependence implies that the DFT MD have to be performed for very large sizes to pinpoint the low-temperature limit of the $b c c$ stability which is impossible. While exact sizes where the size convergence of DFT MD will be reached might be different from those of the EAM MD, the similarity of size convergence at high temperature suggests that very large sizes are needed similar to those we used in EAM MD.

To check whether the free energy of the $b c c$ phase is affected by its size, we performed two-phase simulation of melting where the solid and liquid are placed in one computational cell forming a common interface [45-47]. Two sizes were explored, 1024 atoms $b c c$ structure and 2,000,000 $b c c$ structure (the liquid part of the computational cell was equal to the solid part size). The smaller system solidified at $7240 \mathrm{~K}$ and melted at $7250 \mathrm{~K}$. The larger system solidified at $7260 \mathrm{~K}$ and melted at $7268 \mathrm{~K}$. We see that the larger size increases the melting temperature but the impact is minor. As soon as the structure is stabilized dynamically and does not collapse to a close-packed structure, the free energy remains pretty constant.

We calculated the free energy of liquid, $b c c$, and $h c p$ phases at a number of pressures between 60 and $360 \mathrm{GPa}$ as a function of temperature using the EAM potential [10]. At all these pressures the $h c p$ free energy is lower than the free energy of $b c c$. The melting curve of the hcp phase is close to the melting curve computed previously [10]. This suggests that the free energies computed correctly for the EAM model. It was demonstrated [18] that electronic entropy substantially 

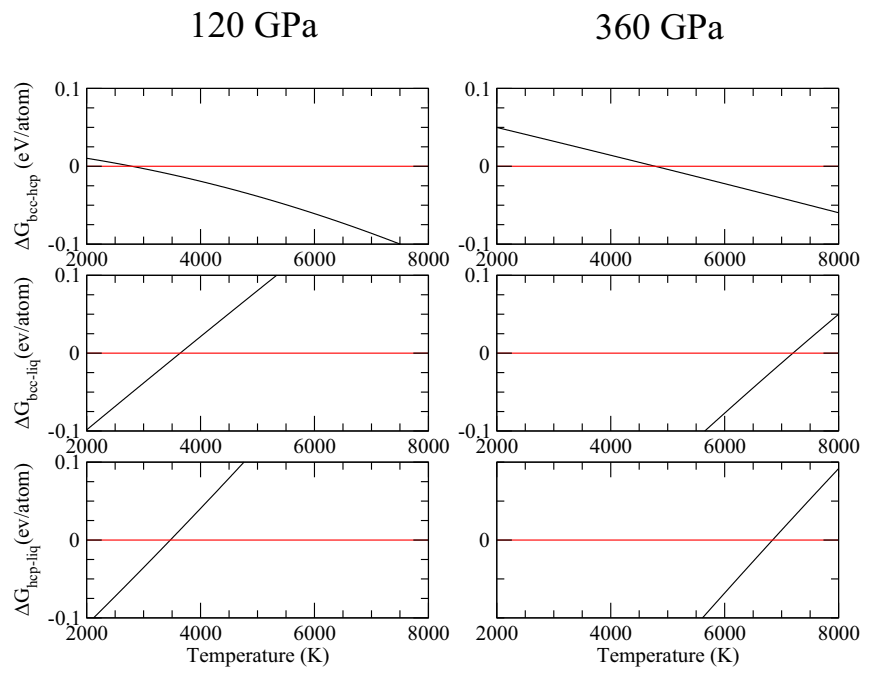

FIG. 5. Difference of Gibbs free energies between the $b c c, h c p$, and liquid phases of iron (as indicated on the axes) as a function of temperature and pressures of $120 \mathrm{GPa}$ (left column) and $360 \mathrm{GPa}$ (right column).

affects the free-energy difference. At the same time, the EAM model does not take electronic entropy into account explicitly. This is a major problem of the EAM model. Even with the potential that takes into account the temperature effect as, for example, suggested by Ackland [48], the system is not capable of taking into account the effect of electron degrees of freedom on the free energy. We calculated the electronic entropy using package VASP at the pressures 120 and $360 \mathrm{GPa}$ for the liquid, bcc, and hcp phases in the NPT ensemble at the temperatures 4000 and $7000 \mathrm{~K}$ correspondingly. Figure 5 shows the free-energy difference for $b c c$-hcp, bcc-liquid, and $h c p$-liquid with electronic entropy correction. We note first that the melting temperature of the EAM $h c p$ if corrected for the electronic entropy becomes equal to $6800 \mathrm{~K}$ at $360 \mathrm{GPa}$. The latest assessment of the $h c p$ melting temperatures [49] provides the range of temperatures between 5400 and $6345 \mathrm{~K}$ at the pressure of $330 \mathrm{GPa}$ (the pressure at the boundary between solid IC and the liquid outer core). The derivative of the melting temperature on pressure varies between 8 and $11 \mathrm{~K}$ per $1 \mathrm{GPa}$. One can estimate the melting temperature at the pressure of $360 \mathrm{GPa}$ as varying (depending on the source) between 5700 and $6675 \mathrm{~K}$. Given the error bars of about $200 \mathrm{~K}$, the $6800 \mathrm{~K}$ we obtained seems to be at the upper end of the range, yet reasonably close (or even within error bars) to most of the numbers provided in Table 2 of Ref. [49]. This is confirmation that our approach is reasonable. The temperature of the $h c p-b c c$ transition is $4900 \mathrm{~K}$, and the $b c c$ melts at $7190 \mathrm{~K}$. At the pressure of $120 \mathrm{GPa}$ the $h c p$ phase melts at $3450 \mathrm{~K}$ as compares with the $3970 \mathrm{~K}$ [50]. The $h c p-b c c$ transition is at $2800 \mathrm{~K}$, and the $b c c$ phase melts at $3650 \mathrm{~K}$. Note, that the straight line that connects the points of the $h c p-b c c$ transition is in very decent agreement with the experimental data as measured by Hrubiak et al. [25]. Figure 6 summarizes the calculated phase boundaries and provides comparison with existing data. The calculated phase boundaries are shown schematically by straight lines.

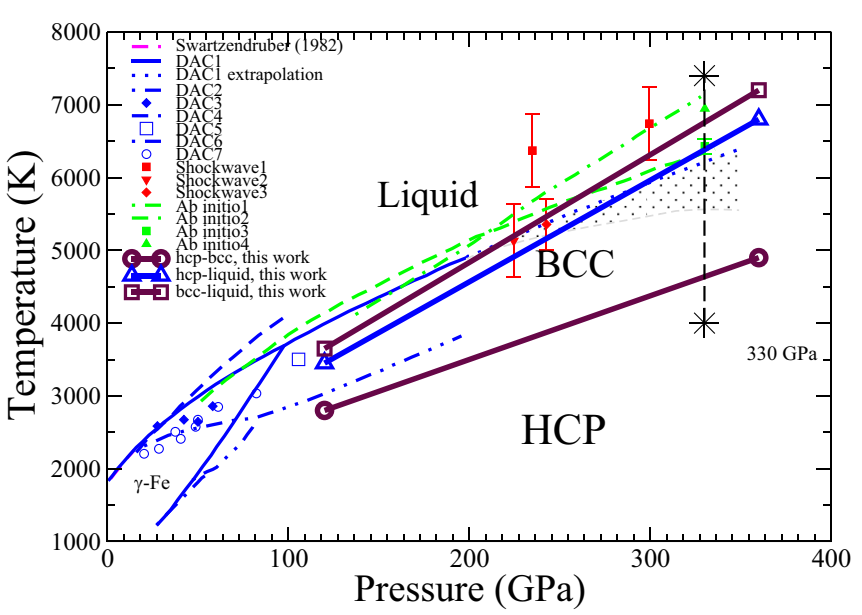

FIG. 6. Iron phase diagram. The new data that shows (schematically) hcp- $b c c$ and bcc-liquid boundaries as calculated in this work (also shown hcp - liquid phase boundary for the metastable liquid and $h c p$ phases) are compared with the previous experimental and theoretical data. The new data are shown in the plot by brown open squares, blue open triangles, and brown open circles. The previous data are as follows. The magenta dashed line is the Fe phase diagram data reported in 1982 [65]. The blue solid line is diamond anvils cell (DAC) data by fast $\mathrm{x}$-ray diffraction, while the blue dotted line represents the extrapolated melting line of $\mathrm{Fe}$ [6]. The blue dash-dotdotted line [58], the filled diamonds [63], the dashed line [66], the open square [61], the dash-dotted line [60], and the open circles [59] are DAC high pressure data. The red filled squares [67], the filled down triangle [62], and the filled diamond [20] represent shockwave compression data. The green dash-dotted [10] and dashed lines [50] represent the Fe melting curve calculated by the MD method, while the green filled square and the filled up triangle indicate other $a b$ initio results $[57,64]$.

\section{DISCUSSION}

The combination EAM MD along with electronic entropy provided by DFT MD provide the melting curve of the $h c p$ phase in good agreement with the melting curve computed using DFT MD [49-51]. The same combination provides the $h c p-b c c$ transition in good agreement with experiment [25]. Therefore, we have good reason to suggest that, indeed, iron transforms to the high-PT $b c c$ phase on heating at high pressure, at least at the pressures of the Earth IC (330-364 GPa). Finally, if we still are not convinced by the new theoretical and experimental data (admittedly scarce, though), then it is the Earth itself telling us what phase of iron is in its center. The exact match of the angles between the slow and fast axes of sound propagation in the Earth IC [31] $\left(54^{\circ}\right)$ and in the $b c c$ phase $[13,32]\left(54.73^{\circ}\right)$ is the ultimate evidence of stability in the IC. One might ask, however, why, despite numerous experiments on iron, so few have actually observed $[14,20,21,24,25]$ the high-PT $b c c$ phase? Why have so many theoretical studies failed to observe the stability of the high-PT $b c c$ phase? As for the theoretical results, the answer is simple. The simulation of the high-PT $b c c$ requires a large number of atoms (at least 1024 and much larger to compute the range of the stability correctly). Practically all of the previous DFT MD studies relied on simulation of a 
much smaller number of atoms; therefore, their results concerning the dynamic and thermodynamic stability of $b c c$ are likely inconclusive [29,51-55] at best. The explanation for the misleading theoretical results comes from the oversymmetrization of small computational $b c c$ cells by PBC. The (110) planes in small cells preserve their entirety despite strong thermal motion. Since the $b c c$ phase is dynamically unstable at low $\mathrm{T}$, the planes slide along each other toward the configurational energy minimum. This process is in action also at high $\mathrm{T}$ if the size of the cell is small. The pressure components in small cells change in a quite correlated way where two components are always equal to each other [53] (for example, $\mathrm{xx}$ and yy components of pressure tensor are equal to each other, while the $\mathrm{zz}$ component changes on its own, and after some time, the $\mathrm{xx}$ and $\mathrm{zz}$ are equal to each other with yy oscillating independently). The cell oscillates between close-packed-like states and that ruins the natural dynamics of the $b c c$ phase. It is no surprise when the configurational energy is higher than that in the close-packed phase (being very close, though) and the entropy is lower than that in the $b c c$ phase (because the dynamics of the motion is destroyed by the small cell size) that the Gibbs free energy of the $b c c$ phase comes out higher than that for the hcp phase. This is, for example, the case with the study in Ref. [52], where a quite small cell of 54 atoms was simulated. The small cell size leads to the appearance of the deviatoric stress that would destroy the $b c c$ structure. This was correctly noted in Ref. [55] where the $b c c$ cell with 150 atoms was simulated. However, the authors of Ref. [55] misinterpreted their own results- they decided if the results with 150 atoms demonstrate the nonzero deviatoric stress, then any size of the cell would demonstrate such a stress. Remarkably, they ignored the results that demonstrated the dynamic stability of large $b c c$ cells [11]. Instead, the authors of Ref. [55] blamed the potential EAM that, as we now know, produces the size dependence of the $b c c$ stability onset, in full agreement with DFT MD. Quite remarkably, both papers $[52,55]$ while performing quite correct simulations-yet for too small cells-completely misinterpreted the obtained data. Needless to say, the results on elastic properties of the $b c c$ phase performed so far using DFT MD might be quite misleading, again because of the small cell size. The authors of Ref. [51] computed melting temperatures of $b c c$ and $h c p$ iron at high pressure using small cells and claimed the close proximity of those melting temperatures. The claim might be correct, but apparently for the wrong reason, because the authors simulated too small cells. The melting temperature of the $h c p$ phase is most likely correct, while the melting temperature of the $b c c$ phase is affected by the instability of the small $b c c$ cell. At the same time, there are quite a few experimental studies that reported no observation of the $b c c$ phase. First, the most apparent reason could be the slow kinetics of the $h c p-b c c$ transition. The $b c c$ phase is stabilized by the entropy. Such stabilization is expected to be slower than the stabilization by the lower enthalpy. It takes time to visit all the accessible states in the $b c c$ structure; besides, the nuclei of the $b c c$ has to be large to fully accommodate all the states. Second, the $b c c$ is a superionic phase; the diffusion of atoms is comparable to that in a liquid. That might make $\mathrm{x}$-ray identification of the $b c c$ structure difficult. It is not impossible that the diffusion will lead to the observation of a diffuse ring along with the crystalline spots, as, for example, was observed in some experiments $[6,56]$. The strongest $b c c$ peak 110 coincides with the 100 peak of $h c p$. The rest of the $b c c$ peaks are considerably less intense because of the thermal motion coupled with superionic diffusion.

It becomes obvious that the whole field is likely affected by the unexpected physics of the $b c c$ iron phase. At the moment, we have very little data on the $b c c$ properties. Therefore, any interpretation of the Earth IC seismic data is limited. New careful experiments and simulations are required to put the interpretation of seismic data on solid ground. Considering that the properties of iron core are tightly connected to the existence of the Earth-protecting magnetic field and that the existence of such a field is considered as the condition for life, the properties of iron predicted in this paper are needed to search for inhabited exoplanets.

\section{CONCLUSIONS}

We demonstrated that the $b c c$ phase becomes stable above about $5000 \mathrm{~K}$ at the pressures of the Earth IC. At lower pressures (above $120 \mathrm{GPa}$ ) the $h c p-b c c$ phase boundary is in close proximity with the melting curve as determined by Boehler [58] and the experimental hcp-bcc phase boundary as measured by Hrubiak et al. [25]. Given that the hcp-liquid phase boundary, calculated before using fully ab initio MD [49], and the one in this paper, calculated using the hybrid approach, are practically identical, we trust that the precision of the $h c p-b c c$ phase boundary determination is similar to that of fully $a b$ initio MD. We note, however, that fully $a b$ initio MD calculation of the $h c p-b c c$ phase boundary at present is technically impractical. Ab initio MD data on the $b c c$ phase of iron at pressures of the Earth core and higher, in the range of SuperEarth pressures, such as elastic constants, equation of state, heat conductivity, viscosity, etc., are urgently needed. Experimentalists should consider the very special nature of the $b c c$ phase.

\section{ACKNOWLEDGMENTS}

Computations were performed using the facilities at the Swedish National Infrastructure for Computing (SNIC) located at NSC in Linköping. We thank the Swedish Research Council (VR) for financial support (Grant No. 2017-03744) and the National Natural Science Foundation of China (NSFC) (Grant No. 11804175). The article was prepared within the framework of the HSE University Basic Research Program. A.B.B. acknowledges support from the Olle Engkvist Byggmästare Foundation. 
[3] L. V. Altshuler, K. K. Krupnikov, B. N. Ledenev, V. I. Zhuchikhin, and M. I. Brazhnik, Zh. Eksp. Teor. Fiz. 34, 874 (1958).

[4] A. M. Dziewonski and D. L. Anderson, Phys. Earth Planet. Inter. 25, 297 (1981).

[5] R. J. Hemley and H.-K. Mao, Int. Geol. Rev. 43, 1 (2001).

[6] S. Anzellini, A. Dewaele, M. Mezouar, P. Loubeyre, and G. Morard, Science 340, 464 (2013).

[7] S. Tateno, K. Hirose, Y. Ohishi, and Y. Tatsumi, Science 330, 359 (2010).

[8] S. J. Turneaure and S. M. Sharma, and Y. M. Gupta, Phys. Rev. Lett. 125, 215702 (2020).

[9] T. Sakai, E. Ohtani, N. Hirao, and Y. Ohishi, Geophys. Res. Lett. 38, L09302-1-5 (2011).

[10] A. B. Belonoshko, R. Ahuja, and B. Johansson, Phys. Rev. Lett. 84, 3638 (2000).

[11] A. B. Belonoshko, R. Ahuja, and B. Johansson, Nature (London) 424, 1032 (2003).

[12] A. B. Belonoshko, N. V. Skorodumova, S. Davis, A. N. Osiptsov, A. Rosengren, and B. Johansson, Science 316, 1603 (2007).

[13] A. B. Belonoshko, N. V. Skorodumova, A. Rosengren, and B. Johansson, Science 319, 797 (2008).

[14] A. B. Belonoshko, P. M. Derlet, A. S. Mikhaylushkin, S. I. Simak, O. Hellman, L. Burakovsky, D. C. Swift, and B. Johansson, New J. Phys. 11, 093039 (2009).

[15] A. B. Belonoshko, A. Rosengren, L. Burakovsky, D. L. Preston, and B. Johansson, Phys. Rev. B 79, 220102(R) (2009).

[16] M. Mattesini, A. B. Belonoshko, E. Buforn, M. Ramírez, S. I. Simak, A. Udías, H.-K. Mao, R. Ahuja, Proc. Natl. Acad. Sci. USA 107, 9507 (2010).

[17] A. B. Belonoshko, T. Bryk, and A. Rosengren, Phys. Rev. Lett. 104, 245703 (2010).

[18] A. B. Belonoshko, T. Lukinov, J. Fu, J. Zhao, S. Davis, and S. I. Simak, Nat. Geosci. 10, 312 (2017).

[19] A. B. Belonoshko, J. Fu, T. Bryk, S. I. Simak, and M. Mattesini, Nat. Commun. 10, 2483 (2019).

[20] J. M. Brown and R. G. McQueen, J. Geoph. Res. 91, 7485 (1986).

[21] J. M. Brown, Geophys. Res. Lett. 28, 4339 (2001).

[22] L. Dubrovinsky, N. Dubrovinskaia, O. Narygina, I. Kantor, A. Kuznetzov, V. B. Prakapenka, L. Vitos, B. Johansson, A. S. Mikhaylushkin, S. I. Simak, and I. A. Abrikosov, Science 316, 1880 (2007).

[23] A. S. Mikhaylushkin, S. I. Simak, L. Dubrovinsky, N. Dubrovinskaia, B. Johansson, and I. A. Abrikosov, Phys. Rev. Lett. 99, 165505 (2007).

[24] Y. Ping, F. Coppari, D. G. Hicks, B. Yaakobi, D. E. Fratanduono, S. Hamel, J. H. Eggert, J. R. Rygg, R. F. Smith, D. C. Swift, D. G. Braun, T. R. Boehly, and G. W. Collins, Phys. Rev. Lett. 111, 065501 (2013).

[25] R. Hrubiak, Y. Meng, and G. Shen, arXiv:1804.05109 (2018).

[26] X. Sha and R. E. Cohen, Geophys. Res. Lett. 37, L10302-1-5 (2010).

[27] A. Cao, B. Romanowicz, and N. Takeuchi, Science 308, 1453 (2005).

[28] G. Steinle-Neumann, L. Stixrude, R. E. Cohen, and O. Gälseren, Nature 413, 57 (2001).

[29] P. Modak, A. K. Verma, R. S. Rao, B. K. Godwal, L. Stixrude, and R. Jeanloz, J. Phys.: Condens. Matter 19, 016208 (2006).
[30] D. Antonangeli, F. Occelli, H. Requardt, J. Badro, G. Fiquet, and M. Krisch, Earth Planet. Sci. Lett. 225, 243 (2004).

[31] J. Stephenson, H. Tkalčić, and M. Sambridge, J. Geoph. Res. 126, e2020jb020545 (2021).

[32] M. Mattesini, A. B. Belonoshko, H. Tkalčić, E. Buforn, A. Udias, and R. Ahuja, Sci. Rep. 3, 2096 (2013).

[33] J. P. Perdew, J. A. Chevary, S. H. Vosko, K. A. Jackson, M. R. Pederson, D. J. Singh, and C. Fiolhais, Phys. Rev. B 46, 6671 (1992).

[34] P. E. Blöchl, Phys. Rev. B 50, 17953 (1994).

[35] G. Kresse and D. Joubert, Phys. Rev. B 59, 1758 (1999).

[36] G. Kresse and J. Furthmüller, Phys. Rev. B 54, 11169 (1996).

[37] A. P. Sutton and J. Chen, Philos. Mag. Lett. 61, 139 (1990).

[38] S. Plimpton, J. Comput. Phys. 117, 1 (1995).

[39] R. Freitas, M. Asta, and M. de Koning, Comput. Mater. Sci. 112, 333 (2016).

[40] R. Paula Leite and M. de Koning, Comput. Mater. Sci. 159, 316 (2019).

[41] R. Paula Leite, R. Freitas, R. Azevedo, and M. de Koning, J. Chem. Phys. 145, 194101 (2016).

[42] R. Paula Leite, P. A. Santos-Florez, and M. de Koning, Phys. Rev. E 96, 032115 (2017).

[43] M. de Koning, A. Antonelli, and S. Yip, J. Chem. Phys. 115, 11025 (2001).

[44] A. J. Schultz, S. G. Moustafa, and D. A. Kofke, Sci. Reports 8, 7295 (2018).

[45] A. B. Belonoshko, Geochim. Cosmochim. Acta 58, 4039 (1994).

[46] A. B. Belonoshko, Amer. Min. 81, 303 (1996).

[47] A. B. Belonoshko, Geochim. Cosmochim. Acta 59, 1883 (1995).

[48] G. J. Ackland, J. Phys.: Conf. Ser. 402, 012001 (2012).

[49] T. Sun, J. P. Brodholt, Y. Li, and L. Voĉadlo, Phys. Rev. B 98, 224301 (2018).

[50] D. Alfè, G. D. Price, and M. J. Gillan, Phys. Rev. B 65, 165118 (2002).

[51] J. Bouchet, S. Mazevet, G. Morard, F. Guyot, and R. Musella, Phys. Rev. B 87, 094102 (2013).

[52] L. Voĉadlo, D. Alfè, M. J. Gillan, I. G. Wood, J. P. Brodholt, and G. D. Price, Nature (London) 424, 536 (2003).

[53] A. B. Belonoshko, S. Arapan, and A. Rosengren, J. Phys.: Condens. Matter 23, 485402 (2011).

[54] W. Luo, B. Johansson, O. Eriksson, S. Arapan, P. Souvatzis, M. I. Katsnelson, and R. Ahuja, Proc. Natl. Acad. Sci. USA 107, 9962 (2010).

[55] B. K. Godwal, F. Gonzalez-Cataldo, A. K. Verma, L. Stixrude, and R. Jeanloz, Earth Planet. Sci. Lett. 409, 299 (2015)

[56] G. Y. Shen, H.-K. Mao, R. J. Hemley, T. S. Duffy, and M. L. Rivers, Geophys. Res. Lett. 25, 373 (1998).

[57] D. Alfè, Phys. Rev. B 79, 060101(R) (2009).

[58] R. Boehler, Nature (London) 363, 534 (1993).

[59] J. M. Jackson, W. Sturhahn, M. Lerche, J. Zhao, T. S. Toellner, E. E. Alp, S. V. Sinogeikin, J. D. Bass, C. A. Murphy, and J. K. Wicks, Earth Planet. Sci. Lett. 362, 143 (2013).

[60] T. Komabayashi, Y. Fei, Y. Meng, and V. B. Prakapenka, Earth Planet. Sci. Lett. 282, 252 (2009).

[61] Y. Ma, M. Somayazulu, G. Shen, H. Mao, J. Shu, and R. J. Hemley, Phys. Earth Planet. Inter. 143, 455 (2004). 
[62] J. H. Nguyen and N. C. Holmes, Nature (London) 427, 339 (2004).

[63] G. Shen, V. B. Prakapenka, M. L. Rivers, and S. R. Sutton, Phys. Rev. Lett. 92, 185701 (2004).

[64] E. Sola and D. Alfè, Phys. Rev. Lett. 103, 078501 (2009).
[65] L. J. Swartzendruber, Bull. Alloy Phase Diagrams 3, 161 (1982).

[66] Q. Williams, R. Jeanloz, J. Bass, B. Svendsen, and T. J. Ahrens, Science 236, 4798 (1987).

[67] C. S. Yoo, N. C. Holmes, M. Ross, D. J. Webb, and C. Pike, Phys. Rev. Lett. 70, 3931 (1993). 